\title{
Living donor liver retransplantation for primary non-function of liver graft following multivisceral transplantation in a pediatric patient
}

\author{
Shin Hwang ${ }^{1}$, Dae-Yeon $\mathrm{Kim}^{2}$, Jung-Man Namgoong ${ }^{2}$, Kyung-Mo $\mathrm{Kim}^{3}$, Seak Hee $\mathrm{Oh}^{3}$, \\ $\mathrm{Ki}-\mathrm{Hun} \mathrm{Kim}^{1}$, Chul-Soo Ahn ${ }^{1}$, Hyunhee Kwon ${ }^{2}$, Yu Jeong $\mathrm{Cho}^{2}$, and Yong Jae Kwon ${ }^{2}$ \\ ${ }^{1}$ Division of Hepatobiliary Surgery and Liver Transplantation, Department of Surgery, Asan Medical Center, \\ University of Ulsan College of Medicine, ${ }^{2}$ Division of Pediatric Surgery, Department of Surgery, \\ Asan Medical Center, University of Ulsan College of Medicine, \\ ${ }^{3}$ Department of Pediatrics, Asan Medical Center, University of Ulsan College of Medicine, Seoul, Korea
}

\begin{abstract}
Multivisceral organ transplantation (MVOT) includes transplantation of three or more abdominal organs, generally including the small bowel, duodenum, stomach, liver, pancreas, and colon. We here presented the detailed procedures of repeat living donor liver transplantation for primary non-function of the first liver graft following MVOT in a pediatric patient. A 6-year-old girl with chronic intestinal pseudo-obstruction underwent MVOT with 5-year-old donor organs. However, the primary non-function of the liver graft developed, and an emergency living donor liver transplantation operation using a left lateral section graft was performed on the third day after MVOT. The donor was the patient's father. Portal flow interruption induced ischemic congestion of the whole small bowel, thus we used a series of porto-caval shunt to reduce the risk of ischemic splanchnic congestion during recipient hepatectomy and graft implantation. Other surgical procedures were the same as the standardized procedures for left liver graft implantation. The graft-recipient weight ratio was 2.15 . The patient was managed conservatively for 3 months and discharged in an improved condition at 4 months after MVOT. She finally passed away at 22 months after MVOT. We think that our experience will be helpful for surgeons to cope with portal vein clamping-associated splanchnic congestion during liver transplantation and other abdominal surgeries. (Ann Hepatobiliary Pancreat Surg 2020;24:198-202)
\end{abstract}

Key Words: Multivisceral transplantation; Primary non-function; Living donor liver transplantation; Portocaval shunt

\section{INTRODUCTION}

Multivisceral organ transplantation (MVOT) includes transplantation of three or more abdominal organs, generally including the small bowel, duodenum, stomach, liver, pancreas, and colon. ${ }^{1}$ In some patients with renal failure, it is noted that the kidneys can also be included as an en-bloc graft. ${ }^{2}$ With advances in surgical techniques and development of potent immunosuppressive regimens, the patient survival rates following MVOT and intestinal transplantation were improved to $92 \%$ at 1 year and $70 \%$ at 5 years. $^{3}$

However, MVOT is still regarded as a challenging surgery to many transplant surgeons. The initial experience of MVOT may be faced with many technical obstacles, lead- ing to prolongation of operation time and ischemic time. Our colleagues reported a case of pediatric patient who experienced severe post-reperfusion syndrome following implantation of seven abdominal organs and thereafter liver retransplantation due to primary non-function (PNF) of the first liver graft. ${ }^{4}$ It was the same case that we herein reported.

We here presented the detailed procedures of repeat living donor liver transplantation (LDLT) for PNF of the first liver graft following MVOT in a pediatric patient because portal flow interruption induced severe ischemic congestion of the whole small bowel unlike in the usual LDL cases.

Received: March 18, 2020; Revised: March 22, 2020; Accepted: March 23, 2020

Corresponding author: Shin Hwang

Department of Surgery, Asan Medical Center, University of Ulsan College of Medicine, 88 Olympic-ro 43-gil, Songpa-gu, Seoul 05505, Korea Tel: +82-2-3010-3930, Fax: +82-2-3010-6701, E-mail: shwang@amc.seoul.kr

Copyright (C) 2020 by The Korean Association of Hepato-Biliary-Pancreatic Surgery

This is an Open Access article distributed under the terms of the Creative Commons Attribution Non-Commercial License (http://creativecommons.org/ censes/by-nc/4.0) which permits unrestricted non-commercial use, distribution, and reproduction in any medium, provided the original work is properly cited. Annals of Hepato-Biliary-Pancreatic Surgery • pISSN: 2508-5778 - elSSN: 2508-5859 


\section{CASE}

A 6-year-old girl weighing $17 \mathrm{~kg}$, with chronic intestinal pseudo-obstruction was scheduled for MVOT. Four years before, she underwent gastropexy and segmental resection of the transverse colon due to gastric volvulus and congenital megacolon. However, the patient's symptoms did not improve after surgery, thus she was repeatedly hospitalized and maintained on the programs of total parenteral nutrition. She was finally scheduled for MVOT,

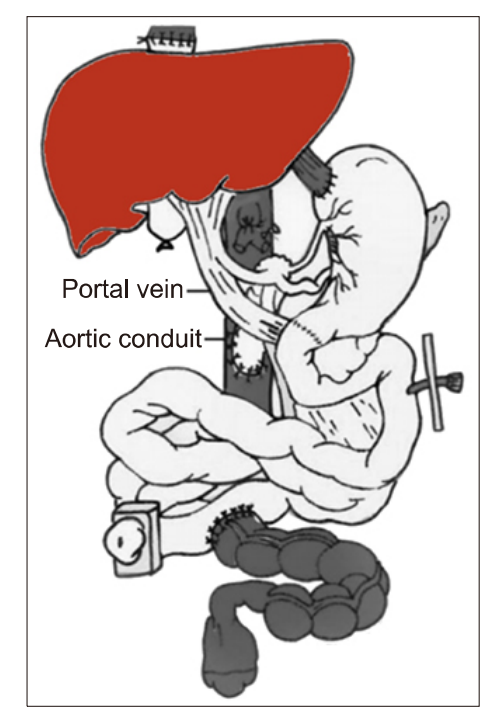

Fig. 1. Schematic illustration of multivisceral organ transplantation. including seven abdominal organs, the liver, spleen, stomach, duodenum, small bowel, colon and pancreas (Fig. 1). The deceased donor was a 5-year-old female weighing 21 $\mathrm{kg}$ suffered from medulloblastoma. Routine surgical procedures for MVOT were performed (Fig. 2), but the total ischemic time was prolonged. Just after graft reperfusion, severe postreperfusion syndrome developed, which was recovered through intensive intraoperative support. ${ }^{4}$

Soon after the MVOT operation, serum aspartate transaminase (AST) and alanine transaminase (ALT) levels increased rapidly: immediate postoperative AST 2,108 IU/L and ALT 2,351 IU/L; and the second day, AST 7,300 IU/L and ALT 6,400 IU/L. The patient's liver function was rapidly deteriorated, thus being diagnosed of PNF of the liver graft.

An emergency LDLT operation using a left lateral section graft was performed on the third day after MVOT. The donor for the patient was her father. After laparotomy, it is noted that the first liver graft was discolored (Fig. 3A). The hepatic hilum was dissected first. Just after clamping of the main portal vein, the whole small bowel became markedly discolored (Fig. 3B), because there was no outflow pathway of the splanchnic blood flow except the portal vein. A $10 \mathrm{~cm}$-long segment of cold-stored iliac vein allograft was anastomosed to the retrohepatic inferior vena cava (IVC) and a porto-caval shunt was made (Fig. 3C). Under this porto-caval bypass, the recipient hepatectomy
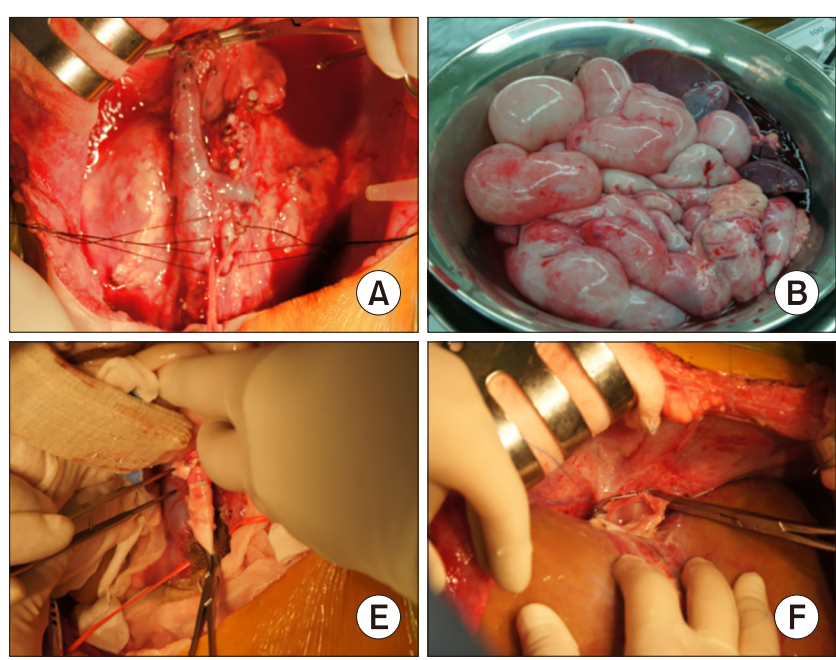
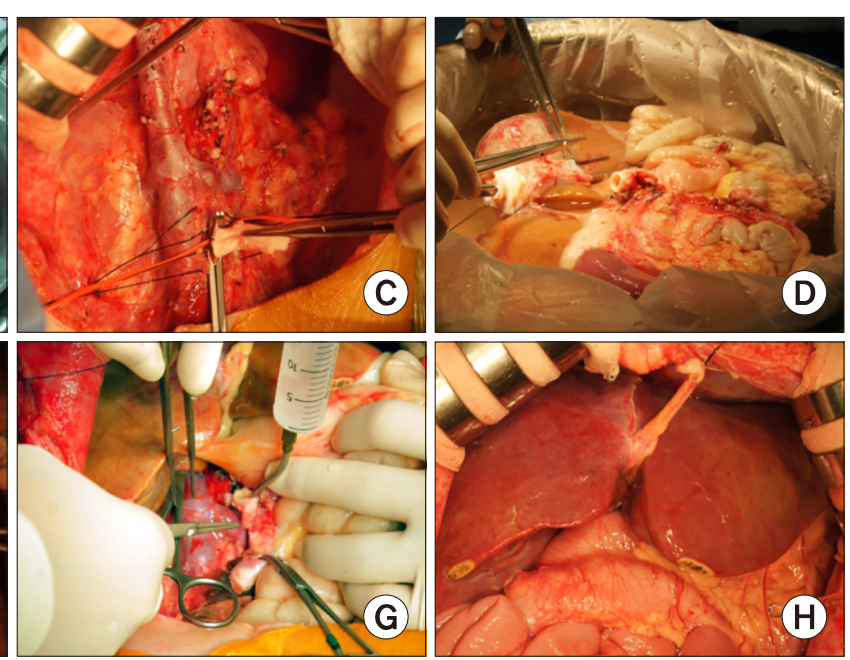

Fig. 2. Intraoperative photographs of multivisceral organ transplantation. (A, B) Abdominal organs were completely removed. (C) An aortic conduit is anastomosed to the recipient aorta. (D) Donor multivisceral organs were prepared at the back table. (E) The graft aorta stump was reconstructed at the aortic conduit. (F) The graft inferior vena cava was reconstructed. (G) The aorta conduit clamp was released. $(\mathrm{H})$ The multivisceral organs were reperfused. 

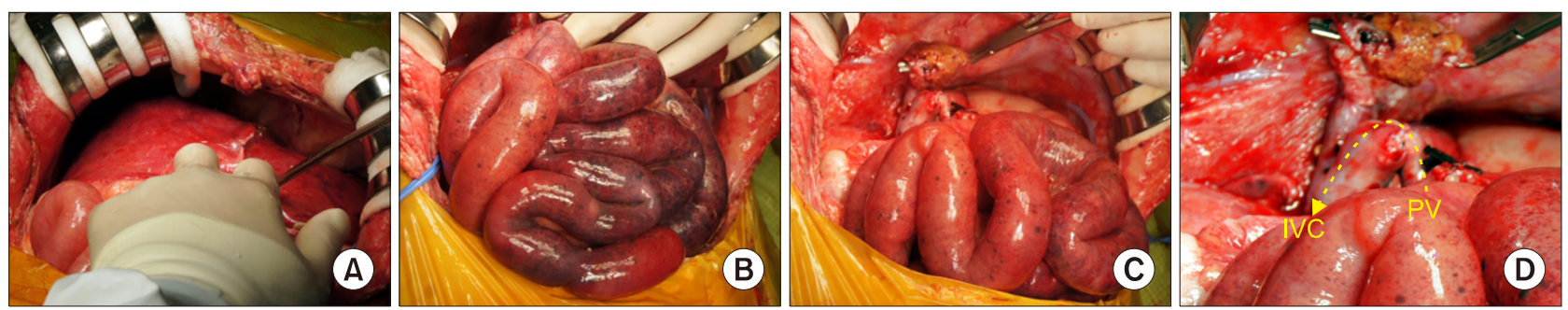

Fig. 3. Intraoperative photographs of recipient hepatectomy during repeat living donor liver transplantation. (A) The first liver graft was discolored due to primary non-function. (B) The whole small bowel became discolored shortly after clamping of the main portal vein. (C, D) A cold-stored iliac vein allograft was anastomosed to the retrohepatic inferior vena cava (IVC) and the recipient portal vein (PV) stump, by which a porto-caval shunt was made.
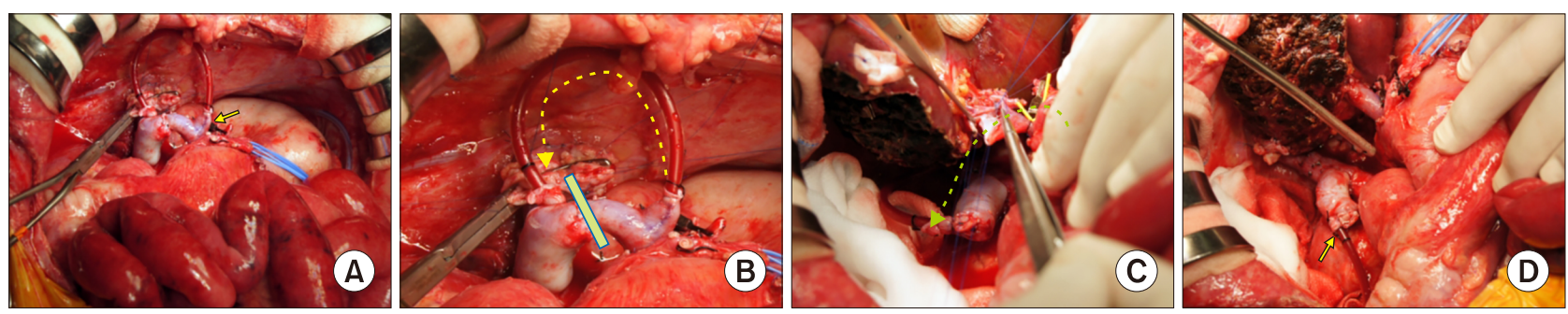

Fig. 4. Intraoperative photographs of graft implantation during repeat living donor liver transplantation. (A) The left portal vein branch was isolated and a catheter was connected between the left portal vein stump (arrow) and the connecting part of the porto-caval shunt. (B) When the porto-caval shunt was clamped, the portal vein blood flow was bypassed through a catheter. (C) Portal vein was reconstructed under porto-caval shunt using a catheter (dotted arrow). (D) The disconnected catheter (arrow) was visible after the portal vein reconstruction.
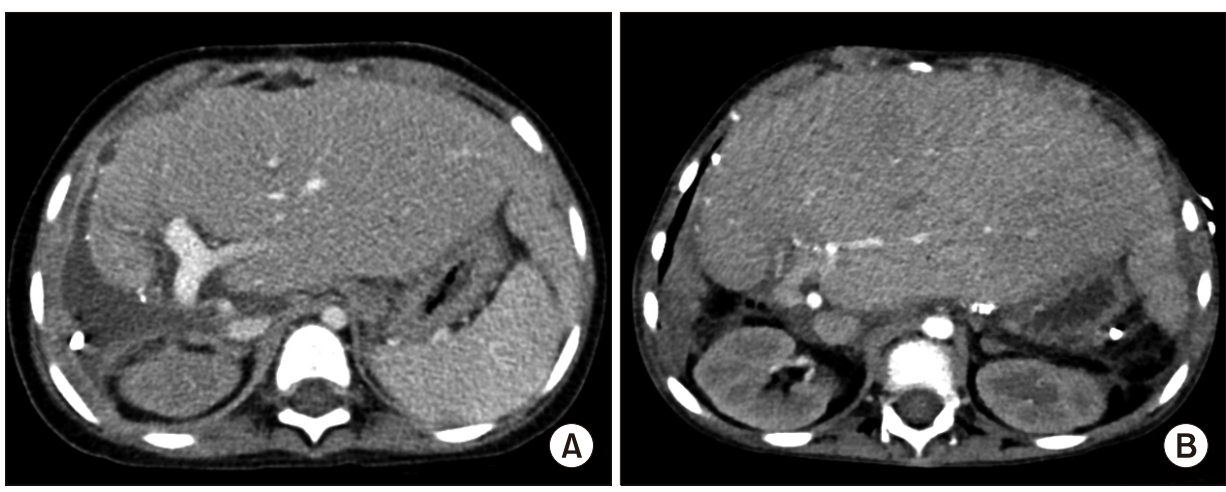

Fig. 5. Posttransplant computed tomography scans. (A) Image taken 1 month after transplantation showed no abnormality of the graft liver. (B) Image taken 20 months after transplantation showed precirrhotic changes of the graft liver.

was performed (Fig. 3D).

At this time, we recognized that portal vein occlusion during portal vein reconstruction would induce ischemic congestion of the small bowel again. The main portal vein was dissected deeply and the right and left portal vein branches were isolated. A $15 \mathrm{~cm}$-long catheter, which was used for usual intravenous fluid injection, was connected between the left portal vein stump and the connecting part of the porto-caval shunt (Fig. 4A). Thereafter, the graft hepatic vein was anastomosed to the recipient hepatic vein orifice under total clamping of the IVC and simultaneous clamping of the aorta jump graft because there was no outflow pathway of splanchnic venous blood flow. Just after graft hepatic vein reconstruction, the neck portion of graft hepatic vein anastomosis was clamped and then the IVC was released. This procedure took about 10 minutes to perform, which was an ischemic time of the small bowels. The connecting portion of the porto-caval shunt was separated (Fig. 4B), and the recipient right portal vein stump was anastomosed to the graft portal vein (Fig. 4C). During this procedure, the intravenous infusion catheter worked as another porto-caval bypass route (Fig. 4D), by 
which the small bowel was no longer congested. Other LDLT procedures were followed including the standard procedures for left graft implantation (Fig. 5A). The graft weight was $365 \mathrm{~g}$, thus the graft-recipient weight ratio was 2.15 .

The patient was managed conservatively for 3 months including 50-day stay in the intensive care unit and was discharged in an improved condition at 4 months after MVOT. She had suffered from repeated episodes of inflammation of the small bowel and colon and acute rejection of the liver (Fig. 5B). She finally passed away at 22 months after MVOT.

\section{DISCUSSION}

This case was the first case of MVOT in Korea, which was performed in October 2011. Many cases of liver transplantation have been performed in our institution, ${ }^{5,6}$ but MVOT has been regarded as a challenging surgery. We presume that the lack of experience on MVOT led to prolongation of total ischemic time, which might result in PNF of the liver graft because the tolerable ischemic time for the liver graft is shorter than the other abdominal organs. $^{7}$

The lack of experience on MVOT also led us to face a difficult situation of severe splanchnic venous congestion because the venous collaterals are completely interrupted thus the portal vein works as the only outflow pathway of splanchnic blood flow. Just after observing severe discoloration of the small bowels shortly after portal vein clamping, we recognized the risk of ischemic congestion-associated damage to the transplanted small bowels at that time.

Our technical knacks to cope with liver retransplantation after MVOT were the adoption of timely porto-caval bypass to minimize the risk of splanchnic congestion. First, we used porto-caval bypass during hilar dissection, in which a large-sized iliac vein conduit was used. Second, since the graft hepatic vein reconstruction required complete occlusion of the IVC, we temporarily clamped the aorta conduit to prevent the incidence of small bowel congestion. It induced total ischemia of the small bowel, but we presumed that ischemic insult is better than ischemia combined with severe venous congestion. Third, we also used a small-caliber porto-caval bypass conduit be- tween the left portal vein branch and the IVC. Because the recipient body size was small, small bypass blood flow less than $100 \mathrm{ml}$ per minutes appeared to be enough to prevent small bowel congestion.

The concept of porto-caval bypass for pediatric LDLT was applied to our recent case with advanced pancreatoblastoma. The 4-year-old patient underwent LDLT operation which was combined with spleen-preserving regional total pancreatectomy and portal vein homograft interposition. The patient received spleen-preserving distal pancreatectomy which was performed first, and then completion regional total pancreatectomy was performed under the superior mesenteric vein-IVC bypass. ${ }^{8}$

In Korea, there is a small but considerable demand for intestinal transplantation and MVOT in pediatric patients. Chang et al. ${ }^{9}$ at the Seoul St. Mary's Hospital reported that 5 children and 10 adults (6 months to 69 years of age) underwent bowel transplantation during 10 years. The primary diseases noted in adults included mesenteric vessel thrombosis in 4 , strangulation in 2 , and visceral myopathy, malignant gastrointestinal stromal tumor, mesenteric lymphangiectasis, and injury in each one. Pediatric cases involved Hirschsprung disease in 2, visceral myopathy in 2, and necrotizing enterocolitis in 1 . The bowel transplantation was performed using living donors in 3 , and deceased donors in 12. The types of transplantation were isolated intestinal transplantation in 14, and modified MVOT in 1.

To our knowledge, this case is the first pediatric case of repeat LDLT following MVOT. We think that our experience will be helpful to cope with portal vein clamping-associated splanchnic congestion during liver transplantation and other abdominal surgeries going forward.

\section{ACKNOWLEDGEMENTS}

The authors express thanks to Professor Jang Moon (Recanati Miller Transplantation Institute, Icahn School of Medicine at Mount Sinai, New York, NY, USA) for his valuable discussion.

\section{CONFLICT OF INTEREST}

No potential conflict of interest relevant to this article was reported. 


\section{ORCID}

Shin Hwang: https://orcid.org/0000-0002-9045-2531

Dae-Yeon Kim: https://orcid.org/0000-0001-6130-1796

Jung-Man Namgoong:

https://orcid.org/0000-0002-9237-7440

Kyung-Mo Kim: https://orcid.org/0000-0001-7896-6751

Seak Hee Oh: https://orcid.org/0000-0002-9672-8877

Ki-Hun Kim: https://orcid.org/0000-0002-4016-0995

Chul-Soo Ahn: https://orcid.org/0000-0002-3844-3646

Hyunhee Kwon: https://orcid.org/0000-0001-6647-9155

Yu Jeong Cho: https://orcid.org/0000-0001-6823-2746

Yong Jae Kwon: https://orcid.org/0000-0001-9490-1229

\section{AUTHOR CONTRIBUTIONS}

Conceptualization: SH, DYK. Data curation: JMN, KMK, SHO. Methodology: KHK, CSA, HK, YJC, YJK. Project administration: SH, DYK. Visualization: SH. Writing - original draft: SH. Writing - review \& editing: SH.

\section{REFERENCES}

1. Spechtenhauser B, Königsraner A, Steurer W, Riedmann B, Pomarolli A, Hörmann C, et al. Multivisceral transplantation. Transplant Proc 1999;31:3175-3176.

2. Loinaz C, Mittal N, Kato T, Miller B, Rodriguez M, Tzakis A. Multivisceral transplantation for pediatric intestinal pseudo-obstruction: single center's experience of 16 cases. Transplant Proc 2004;36:312-313.

3. Abu-Elmagd KM, Costa G, Bond GJ, Soltys K, Sindhi R, Wu $\mathrm{T}$, et al. Five hundred intestinal and multivisceral transplantations at a single center: major advances with new challenges. Ann Surg 2009;250:567-581.

4. Park YS, Oh JY, Hwang BY, Moon Y, Lee HM, Hwang GS. Prolonged post-reperfusion syndrome during multivisceral organ transplantation in a pediatric patient: a case report. Korean $\mathrm{J}$ Anesthesiol 2014;66:467-471.

5. Moon DB, Lee SG, Hwang S, Kim KH, Ahn CS, Ha TY, et al. More than 300 consecutive living donor liver transplants a year at a single center. Transplant Proc 2013;45:1942-1947.

6. Moon DB, Lee SG, Hwang S, Kim KH, Ahn CS, Ha TY, et al. Toward more than 400 liver transplantations a year at a single center. Transplant Proc 2013;45:1937-1941.

7. Cai J. Intestine and multivisceral transplantation in the United States: a report of 20-year national registry data (1990-2009). Clin Transpl 2009:83-101.

8. Namgoong JM, Hwang S, Oh SH, Kim KM, Park GC, Ahn CS, et al. Living donor liver transplantation with total pancreatectomy and portal vein homograft replacement in a pediatric patient with advanced pancreatoblastoma. Ann Hepatobiliary Pancreat Surg 2020;24:78-84.

9. Chang HK, Kim SY, Kim JI, Kim SI, Whang JK, Choi JY, et al. Ten-year experience with bowel transplantation at Seoul St. Mary's Hospital. Transplant Proc 2016;48:473-478. 